\title{
Improved Resource Management through User Aggregation in Heterogeneous Multiple Access Wireless Networks
}

\author{
Leonardo Badia, Member, IEEE, Nicola Bui, Marco Miozzo, Michele Rossi, Member, IEEE, \\ and Michele Zorzi, Fellow, IEEE
}

\begin{abstract}
In this letter we discuss the exploitation of aggregated mobility patterns in mobile networks including heterogeneous multiple access techniques. We advocate the use of knowledge about neighboring devices to create routing groups (RGs) of adjacent nodes in order to optimize radio resource management. Basically, RGs consist of aggregated logical structures which are built and maintained at the application layer. Their use allows decreased signaling overhead between groups of nodes and access points (AP) and, at the same time, improved connectivity, which is achieved through the exploitation of technology diversity and relaying schemes. We illustrate a simple yet effective analytical model, and validate it through accurate simulation results. Finally, we show the effectiveness of the RG approach in terms of resource efficiency, throughput and multiple access performance.
\end{abstract}

Index Terms-Communication system routing, multiaccess communication, personal communication networks, hierarchical systems.

\section{INTRODUCTION}

I $\mathrm{N}$ FUTURE generation networks, we expect a strong synergy among heterogeneous radio resources. This is particularly true for Ambient Networks [1], where environmental information, as well as the co-existence of diverse radio access techniques and network management entities, are exploited to achieve a high level of integration. In this letter we address the resource allocation and routing problem in mobile scenarios, where the geographical information about physical proximity and correlated node mobility patterns is exploited by network protocols, similarly to what discussed in [2]. We discuss the creation of aggregated structures that we name Routing Groups (RGs), which are composed of nodes with similar mobility patterns. As an example of the effectiveness of such an approach, think of multiple users moving together and handing over at the same time between two access points. These users may be aggregated in a RG

Manuscript received April 5, 2007; revised July 18, 2007; accepted September 18,2007 . The associate editor coordinating the review of this paper and approving it for publication was M. Guizani. Part of this work has been published in the Proceedings of the 11th International Workshop on ComputerAided Modeling, Analysis and Design of Communication Links and Networks (CAMAD), 2006. This work has been carried out in the framework of the Ambient Networks Project [1] that is partially funded by the Commission of the European Union. The views expressed in this letter are solely those of the authors and should not be interpreted as necessarily representing the views of their employers, this project, or the European Commission.

L. Badia is with the IMT Lucca Institute for Advanced Studies, Piazza San Ponziano 6, 55100 Lucca, Italy.

N. Bui and M. Miozzo are with Consorzio Ferrara Ricerche (CFR), via Saragat 1, 44100 Ferrara, Italy.

M. Rossi (corresponding author) and M. Zorzi are with the University of Padova, Dept. of Information Engineering (DEI), via Gradenigo 6B, 35131 Padova, Italy (e-mail: rossi@dei.unipd.it). M. Rossi and M. Zorzi are also with Consorzio Ferrara Ricerche.

Digital Object Identifier 10.1109/TWC.2008.070362. so that a single message (to the RG leader) needs to be exchanged to successfully accomplish the handover procedure, instead of using a dedicated transmission for each terminal. Another advantage of aggregating devices is the reduction of interfering messages exchanged by neighboring nodes. RGs can in fact be exploited to locally improve the coordination in the data transmission to and from the RG leader. However, these benefits do not come for free, as RGs also need some signaling to be properly set up and maintained [3].

For these reasons, a quantitative evaluation of whether, and to what extent, grouping network terminals in aggregated structures is beneficial, is an important topic for heterogeneous network resource management. Previous work [4]-[7] on device aggregation in wireless networks primarily proposed practical techniques to increase transmission efficiency by means of this rationale. Instead, we investigate how much terminal aggregation can improve the performance from a theoretical standpoint. This considerably differentiates our work, as we neither propose nor compare efficient techniques to aggregate mobile terminals, but we rather evaluate the general paradigm of terminal aggregation. Indeed, all the aforementioned papers can be seen as particular cases of our analysis.

Moreover, we consider heterogeneity among access technologies, which is a very limitedly explored topic in the existing literature. An exception in this sense is represented by [8], where the only case with two access technologies (one short-ranged and one long-ranged) can be found. We take instead a more general approach where any number of technologies, and with general characteristics, can be framed. We presented a preliminary purely analytical investigation in [9], where the RG effectiveness was theoretically shown as a function of various parameters including the density of RGs, the number and type of radio technologies and their energy consumption. Our goal here is to validate these findings through accurate simulation results, where we relax all the restrictive assumptions made in [9] about channel propagation and radio access technologies. The results in this letter show a generally good agreement between theory and simulation. Quantitative differences are however present. They will be discussed and explained in light of physical effects which are taken into account in the simulator and that, for complexity reasons, are difficult to model analytically.

This letter is organized as follows: in Section II we discuss in short the structure, the design philosophy and the capabilities of the simulator. Section III summarizes the analytical framework. In Section IV we compare numerical and analytical results and in Section V we draw our conclusions. 


\section{DESCRIPTION OF THE SIMULATION ENVIRONMENT}

This section gives a short description of our event-driven network simulator for heterogeneous wireless systems called ANEMURAS (Ambient NEtworks MUlti-Radio Architecture Simulator). This tool was developed within the Ambient Networks project [1], and was specifically designed to model a multi-technology mobile and wireless communication scenario including mobile users and fixed access points (APs). Node mobility, wireless channel variability and inter-user/system interference are explicitly and accurately accounted for. Each device (or node) in the simulator can be mobile or static, and behave as a user or an AP. The main goal in designing the simulator structure was to obtain a truly modular and scalable tool, where physical and upper layers are fully decoupled and interchangeable. This allows, for example, the use of the same physical channel module for different radio technologies, which is in charge of all calculations related to timing, power levels, interference and synchronization. Next, we discuss a few more features of the simulator.

Topology: Access points can be either deterministically or randomly placed at the beginning of the simulation and can be either static or on the move. The same applies to mobile users. The only difference between APs and user terminals is that APs may have a high speed path connecting them, e.g., through a wired backbone.

Channel Module: The channel is modeled accounting for path loss, shadowing and multi-path fading phenomena and using their product as the link gain which is subsequently associated with each transmission link. Path loss is implemented according to the well known Hata model [10]: if $P_{t x}$ is the transmitted power, the path loss is calculated as $P_{t x} / P_{r x}=K d^{\beta}$, where $d$ is the distance separating the two communicating entities, whereas $K$ and $\beta$ are constants. Shadowing is accounted for according to Gudmunson's model [10], and multi-path fading is implemented for each link through a Jakes simulator with a programmable number of oscillators [10], [11]. The channel module calculates link gains as the simulation time evolves, whereas a further entity, called physical module, processes the channel gain matrix and user transmission powers to derive intra- and inter-system interference metrics.

Physical Module: The physical module takes as input the channel gain matrix (see channel module above) and transmission powers and returns Signal to Interference plus Noise Ratio (SINR) metrics for each active link. Let $\mathcal{N}$ be the set of nodes in the network and consider a specific user $j$, which wants to receive a data flow from user $i$. The SINR for user $j$ at time $t$ is:

$$
\gamma_{i j}(t)=\frac{S_{i j} P_{t x}^{i}(t) g_{i j}(t)}{\sum_{k \in \mathcal{N}, k \notin\{i, j\}} \alpha_{k j} P_{t x}^{k}(t) g_{k j}(t)+N_{o} B}
$$

where $\gamma_{i j}(t)$ is the SINR experienced by user $j$ at time $t$ and associated with the communication $i \rightarrow j, g_{k j}(t)$, $k, j \in \mathcal{N}, k \neq j$ is the link gain associated with the wireless link connecting node $k$ to node $j, P_{t x}^{i}(t)$ is the transmission power used at time $t$ by user $i, N_{o}$ is the white noise power spectral density and $B$ the transmission bandwidth. $S_{i j}$ is the spreading gain which depends on the specific CDMA codes used in the wireless technology under consideration. In IEEE 802.11b, $S_{i j}=1$, whereas $S_{i j}=S F$ for UMTS, where $S F$ is the spreading factor used by terminal $i[12] . \alpha_{k j}$ is a parameter that we use, at user $j$, to model the fraction of the received power from user $k$ which interferes with transmission $i \rightarrow j$. Observe that $\alpha$ can be used to model interference between non-orthogonal frequency channels (e.g., IEEE 802.11), nonorthogonal spreading codes (e.g., in asynchronous CDMA) or between different systems (e.g., IEEE 802.11 and IEEE 802.15.4).

Other features: The current version of the simulator supports IEEE802.11b (multi-rate transmission and distributed coordination function, DCF), IEEE802.15.4 (ZigBee) and UMTS radio technologies [12], [13]. These are accurately modeled for what concerns physical, link, and MAC layers. For routing, we implemented a modified version of Dynamic Source Routing (DSR), see Section IV. In the simulator both independent and group mobility behaviors are supported, according to the model described in [14]. Data can be generated according to Poisson, continuous and periodic packet generators.

\section{AnAlytical MODEL}

In this section we present the simplified analytical model that we use in this paper and its key assumptions. A similar model was also used in [9].

General assumptions: We consider an Ambient Network where a number of APs and users coexist. We assume that each user requires a separate flow and all flows have the same bitrate $B_{U}$. The extension to different bit-rates is possible with minimal modifications. Both APs and users have a number of different radio technologies which are described by the indices $1,2, \ldots, J$, where technologies are sorted according to their transmission energies. That is, $E_{i}^{t x} \leq E_{j}^{t x}$ if $i<j$. We define three vectors $\mathbf{E}^{t x}=\left\{E_{1}^{t x}, E_{2}^{t x}, \ldots, E_{J}^{t x}\right\}, \mathbf{E}^{r x}=$ $\left\{E_{1}^{r x}, E_{2}^{r x}, \ldots, E_{J}^{r x}\right\}$ and $\mathbf{r}=\left\{r_{1}, r_{2}, \ldots, r_{J}\right\}$ tracking the energies required to transmit and receive a single bit and the maximum transmission ranges for every radio technology, respectively. We additionally assume that $E_{i}^{t x} \leq E_{j}^{t x}$ implies $r_{i} \leq r_{j}$. Not all nodes offer all radio interfaces and, in general, the set of offered interfaces may differ in different nodes. Here, we assume that an interface of type $i$ is available at a generic node with probability $p_{i}$ and that interfaces are assigned independently to each user. For the topology, we consider that users are independently placed according to a planar Poisson process of intensity $\rho$, i.e., the average number of users within an area $\mathcal{A}$ is given by $\rho \mathcal{A}$, whereas the probability to have exactly $n$ nodes in this area is derived as $\mathcal{P}(n, \mathcal{A})=\left((\rho \mathcal{A})^{n} / n !\right) \exp (-\rho \mathcal{A})$. Access points are placed according to a uniform distribution with density $\rho_{A P}$ and are equipped with all technologies $1,2, \ldots, J$.

Within the analysis, nodes positions and channels are considered to be stationary. These assumptions will be relaxed in the simulation results of Section IV. Finally, we consider an idealized MAC by neglecting interference: in the analysis our only interest is to capture the network connectivity (i.e., the availability of a path between any two nodes, in the connectivity graph). The reception capability of the radio interfaces is modeled through vector $\mathbf{r}$. In fact, once the models for path 
loss, shadowing and decoding sensitivity of each radio $i$ are given, it is possible to calculate the corresponding maximum transmission distance $r_{i}$ as a function of the transmission power used by interface $i$ (see $E_{i}^{t x}$ ). Moreover, given the network topology and the radio interface models, we can find the density $\rho_{i}$ of nodes with an interface of type $i$. Formally: $\rho_{i}=\left[\sum_{n=0}^{\infty} \mathcal{P}(n, \mathcal{A}) \sum_{k=0}^{n} k p_{i}(k \mid n)\right] / \mathcal{A}=p_{i} \rho$, where $\mathcal{A} \in \mathbb{R}^{+}, p_{i}(k \mid n)=\left(\begin{array}{l}n \\ k\end{array}\right) p_{i}^{k}\left(1-p_{i}\right)^{n-k}$.

RG formation: Routing groups can be formed through a distributed approach. That is, users cooperate and exchange data to gain information about their physical surroundings and, at the same time, to measure the benefit of grouping with other devices. This involves the periodic exchange of so called HELLO messages between mobile nodes [15]. In each HELLO, every node can for example include the list of its "stable neighbors", i.e., the nodes that have been in its close proximity long enough. In fact, if movements are correlated, stable nodes are likely going to stay in close proximity of the sending device and are therefore good candidates to be grouped with it.

The goal of our analysis is to keep the evaluation of algorithms for RG formation as general as possible, thus we do not investigate a specific aggregation strategy but we simply assume that the RG formation is possible and can be either activated or not, thus determining different network behaviors. The objective of our investigation is to quantify the potential benefits in terms of connectivity with RGs in place and to weigh them against the energy expenditure required to maintain RGs. We further assume that a leader is elected within each RG, having the special role of handling the data traffic so as to optimize the transmission and the access to the channel for RG members. This can be seen, as in standard clustering algorithms [5], [6] for ad hoc networks, as a way to partially centralize the transmission control thereby enhancing the performance. In order to abstract from the specific clustering techniques that might be used to form RGs, and also from the network topology, we adopt a randomized approach for RG leader selection, where each node is chosen as a RG leader with probability $p_{L}$. This holds both for the analysis and for the simulation results that will be shown later. After the RG leader selection, the group is assumed to be created by neighboring nodes which join the leader. Note that this happens in an ideal way in the analysis (nodes always join their closest RG leader), whereas in the simulation we actually implemented the HELLO message procedure (it might happen that a HELLO packet is lost and, in turn, a node may not detect all terminals in its physical proximity). We assume that every interface $i \in\{1,2, \ldots, J\}$ sends HELLO messages with a period $T_{i}^{h}$ and we refer to $b_{i}^{h}$ as the number of bits composing HELLO packets sent by interfaces of type $i$. Moreover, we consider that all $T_{i}^{h} \mathrm{~s}$ are multiple of a reference time period $\Delta T$ such that $T_{i}^{h}=\xi_{i} \Delta T, \xi_{i} \in \mathbb{N}^{+}, i \in\{1,2, \ldots, J\}$, $\Delta T \in \mathbb{R}^{+}$. If we define the least common multiple (LCM) of all $\xi_{i} \mathrm{~s}$ as $\xi$, then we have that $n_{i}^{h}=\frac{\xi}{\xi_{i}}$ is the number of HELLOs sent by the $i$-th interface in a time period of $\xi \Delta T$ seconds.

Connectivity evaluation: The average distance between two APs is $\bar{d}_{A P}=1 /\left(2 \sqrt{\rho_{A P}}\right)$. Hence, on average each AP is in charge of delivering data to all users placed within a circle of radius $\bar{r}_{A P}=\bar{d}_{A P} / 2$ (we neglect the overlap in circular regions, i.e., the terminals which can be identically covered by more than one AP by arbitrarily assigning them to a single AP). The density of nodes with technology $j$ is still given by $\rho_{j}$. The approach to evaluating the network coverage proceeds by slicing the AP coverage area into $J$ different annuli, where the area of the $j$ th annulus is $\mathcal{A}_{j}=\pi\left(r_{j}^{2}-r_{j-1}^{2}\right)$ and contains the region covered by technology $j$ but not by any of the technologies with lower index (i.e., with lower coverage radius). The average number of users that have to be reached in the $j$ th annulus, $\bar{n}_{j}$, is found according to $\bar{n}_{j}=\rho \mathcal{A}_{j}$. The average number of users $\bar{n}_{j, h}$ in the $j$ th annulus that can be optimally covered by exploiting interface $h$ is therefore found as:

$$
\bar{n}_{j, h}= \begin{cases}\rho \tilde{p}_{h j} \mathcal{A}_{j} & h \geq j \\ 0 & h<j,\end{cases}
$$

where $\tilde{p}_{h j}=p_{h}\left[\prod_{j<\ell<h}\left(1-p_{\ell}\right)\right]$, and $p_{\ell}$ is the probability that a generic user has an interface of type $\ell$. The average number of users which can not be reached by any technology, called in the following uncovered users, depends on whether the RG formation is active or not. In the case RGs are used, the coverage radius required is smaller, so that the coverage generally increases. However, an additional connection between the $\mathrm{AP}$ and the RG leader is required. The analytical evaluation of these two quantities leads to: $\bar{n}_{u}=\pi r_{A P}^{2} \rho-\sum_{j=1}^{J} \sum_{h=1}^{J} \bar{n}_{j, h}$ for what concerns the case where RGs are inactive, and:

$$
\bar{n}_{u}=\pi r_{R G}^{2} \rho-\left(1-\exp \left(-\rho_{A P} \pi r_{J}^{2}\right)\right) \sum_{j=1}^{J} \sum_{h=1}^{J} \bar{n}_{j, h},
$$

when they are active. In both cases, the number of uncovered users per unit area is evaluated as $\bar{n}_{u} /\left(\pi \bar{r}_{A P}^{2}\right)$.

Energy consumption evaluation: To evaluate the energy spent for covering the network, we compare the normalized values per unit area. It is possible to obtain the total value multiplying by $\pi \bar{r}_{A P}^{2}$ where $\bar{r}_{A P}=1 /\left(4 \sqrt{\rho_{A P}}\right)$. In the following, the asterisk indicates normalization to the unit area. We obtained in [9] the following expression for the case where RGs are not active:

$$
\bar{E}_{n o R G}^{*}=\frac{\sum_{h=1}^{J} \sum_{j=1}^{J} \bar{n}_{j, h}\left(E_{j}^{t x}+E_{j}^{r x}\right) B_{U}}{\pi \bar{r}_{A P}^{2}} .
$$

For the case where RGs are present, we have instead that the total energy expenditure consists of three terms: $\bar{E}_{R G}^{*}=$ $\bar{E}_{(a) R G}^{*}+\bar{E}_{(b) R G}^{*}+\bar{E}_{(m) R G}^{*}$, where $\bar{E}_{(a) R G}^{*}$ is the energy required to transmit the flow from the AP to the RG leader (see [9] for its detailed analysis), $\bar{E}_{(b) R G}^{*}$ is required to deliver the flow from the leader to the nodes, and finally $\bar{E}_{(m) R G}^{*}$ is the energy to maintain the RG structure. The normalized value $\bar{E}_{(b) R G}^{*}$ is identical to $\bar{E}_{n o R G}^{*}$ (the difference is in the fact that the area of a RG is usually smaller than that covered by an AP, but their evaluation is identical as we do not model power control). Thus, with respect to the case where RGs are not present, the energy expenditure has two more terms. The energy spent to maintain the RG structures over an area $\mathcal{A}$ in 
a time period of $\xi \Delta T$ seconds can instead be derived as:

$$
\begin{array}{r}
\bar{E}_{(m) R G}(\mathcal{A}, T=\xi \Delta T)=\sum_{j=1}^{J} \sum_{n=1}^{\infty} \mathcal{P}(n, \mathcal{A}) \times \\
\times \sum_{k=1}^{n}\left\{k p_{j}(k \mid n) b_{j}^{h} n_{j}^{h}\left[E_{j}^{t x}+E_{j}^{r x} \varepsilon_{j}\right]\right\},
\end{array}
$$

where $\varepsilon_{j}$ is the mean number of nodes receiving the HELLO message sent by a given sending node and using interface $j$ (assuming that HELLO packets are only decoded by the node neighbors whose distance is less than or equal to $\bar{r}_{R G}$ ), i.e.:

$$
\varepsilon_{j}=\sum_{n=2}^{\infty} \mathcal{P}\left(n, \pi \min \left(r_{j}, \bar{r}_{R G}\right)^{2}\right) \sum_{k=1}^{n}(k-1) p_{j}(k \mid n) .
$$

The normalized value of the cost to create and maintain RG structures is therefore derived as:

$$
\bar{E}_{(m) R G}^{*}=\frac{\bar{E}_{R G}(\mathcal{A}, \xi \Delta T)}{\mathcal{A} \xi \Delta T} .
$$

\section{VALidation of the ANALYsis}

In this section, we present simulation results to validate the above analysis while also providing additional insights. We consider a network scenario with two radio access technologies: IEEE802.11b and UMTS. User devices move within a simulation area of $160 \times 160 \mathrm{~m}^{2}$, with speeds uniformly distributed in $[0.5,2] \mathrm{m} / \mathrm{s}$ (e.g., typical pedestrian scenario). The density $\rho$ of the mobile nodes is chosen in [0.001,0.01]. Mobility patterns are generated according to a random way point mobility model. We consider a single AP, placed at the center of the simulation area and owning both technologies. Exactly $20 \%$ of the mobile devices own both wireless technologies, whereas the remaining $80 \%$ of the population is equipped with IEEE $802.11 \mathrm{~b}$ only. As above, we consider two different access strategies: with and without RGs. In the former case (RGs), each user can access the AP either relaying its data to an in-range RG leader or by direct transmission to an in-range AP. Moreover, each node decides between the two previous options by minimizing the number of hops required to deliver its data to the selected access point. In case the user has to decide between two paths having the same number of hops the choice is driven by the available bandwidth. RG leaders are the only nodes which are allowed to aggregate traffic and act as relays, by therefore providing coverage extension through multi-hop routing. RG leaders are elected at random at the beginning of the simulation with probability $p_{L}$ among the users having both technologies. General RG creation strategies can be included in our framework by properly tuning $p_{L}$. Note that in the no RG case relaying is not permitted and a mobile device is connected to the AP if and only if the AP is directly reachable through at least one of the radio technologies owned by the user. On the other hand, in the presence of RGs, a user which does not have a direct connection with any AP can still exploit the relaying functionalities of an in-range RG leader, if present. As will be shown next, this improves connectivity performance. The UMTS network covers the whole simulation area, whereas the IEEE $802.11 \mathrm{~b}$ technology roughly covers half of the simulation area. All users generate uplink traffic (i.e., users $\rightarrow$ AP) at

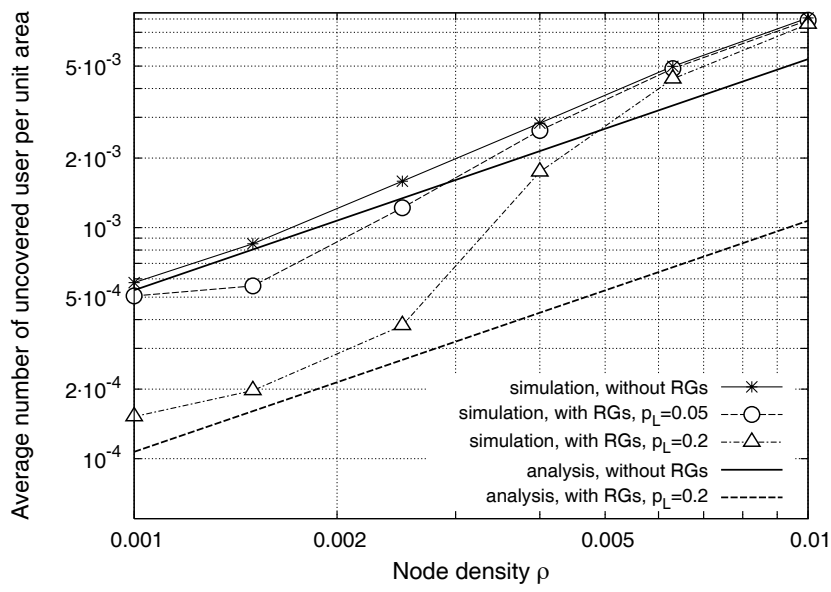

Fig. 1. Average number of uncovered users per unit area as a function of the node density $\rho$.

the rate of one packet per second. Packets are 512 bytes long. Users' traffic is exploited, in part, for the establishment and maintenance of the routes to get to the AP: we modified the DSR protocol in such a way that only RG leaders and APs can relay data traffic.

Next, we report accurate simulation results obtained with the multi-technology simulator presented in Section II. In Fig. 1 we plot the density of uncovered users for both scenarios (with and without RGs). As expected, and in accordance with the analysis, the case without RGs gives the worst performance in terms of connectivity. However, note some deviations from the theoretical investigation. In fact, the analytical curves show a probability of being uncovered around 5 times lower in the case with RGs (even though it is not shown, a similar gain is observed for $p_{L}=0.05$ ). Instead, simulation results always indicate a larger number of uncovered nodes. This is due to a more precise modeling of the propagation scenario, which no longer consists of circular areas. Thus, the analysis is optimistic in estimating the connectivity performance by means of a simplified transmission model. Moreover, the performance of the RG case further degrades as the node density $\rho$ increases. The main reason for this phenomenon is that mutual interference among nodes (neglected in the analysis) strongly increases with the node density, therefore limiting the communication exchanges. This happens for the connections towards the AP, and is in fact the main reason for the increase of all the curves when the node density increases, but also for the inter-RG communications. When $\rho \approx 0.01$, both cases with and without RGs become similar, because the strongest limitation to the connectivity is given by interference. In fact, if the density of transmitters is high, the presence of strong interference is unavoidable, and therefore all terminals, AP, RG members or RG leaders, suffer a performance degradation. In such a case no relaying strategy can help to increase connectivity, which is constrained by the network capacity itself. As a side remark, the operating region where a high number of nodes per unit area is active should be avoided as the performance is dominated by interference and is usually too bad to be considered acceptable. In this case, it would be beneficial to decrease the density of active 


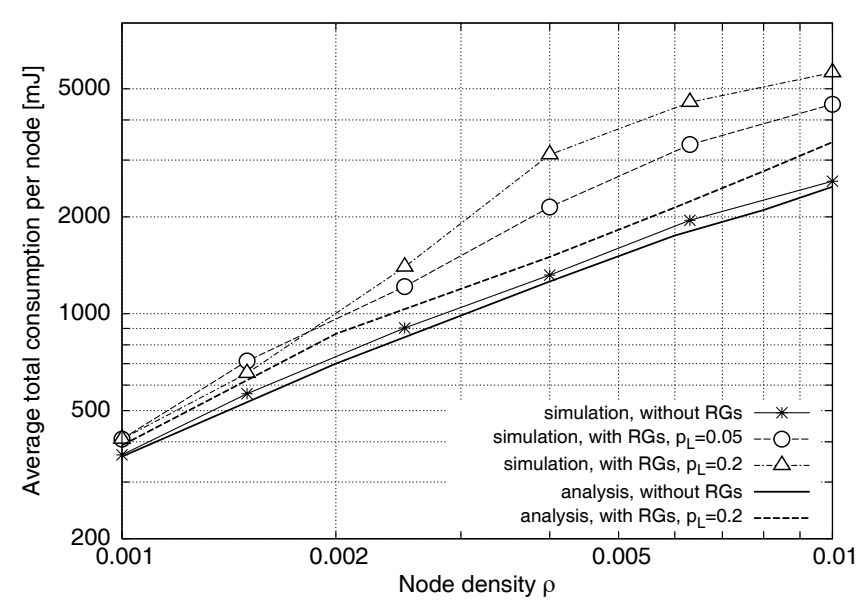

Fig. 2. Average energy consumption per node as a function of the node density $\rho$.

nodes in some way, e.g., by turning off, or excluding from the network, or moving to another portion of the spectrum, some of the terminals. This corresponds to decreasing $\rho$ and moving the operating point of the network so that it is advantageous to use RGs.

Fig. 2 analyzes the energy expenditure of the nodes. The theoretical results are satisfactorily confirmed, although there is always an additional term due to the interference between parallel transmissions, which is not considered in the analysis. Also, in the RG case not only is the consumption higher in absolute terms, but the increase due to taking interference into consideration is also higher, because in the RG case there are more transmitting sources and thus more interference.

The last two figures show how the investigation through simulation results can be useful to analyze in more depth some aspects which were not captured by the analysis as they were not modeled. In Figs. 3 and 4, we report the energy expenditure per correctly delivered packet and the average throughput per node, respectively. It is worth observing that for small to moderate densities $(\rho \leq 0.003$ in the reported graphs) the RG solution leads to better performance in terms of both energy expenditure and throughput. For these densities the aggregation of terminals in close proximity and the exploitation of RG leaders as the relay nodes towards the APs is actually a good strategy. In fact, this makes it possible to exploit the technology diversity offered by RG leaders and, at the same time, to better distribute the traffic among available wireless technologies. However, for very dense networks relaying is less useful as in this case the high interference causes congestion and RG leaders become unable to forward data traffic.

Again, from Fig. 4 we can observe that the maximum improvement is reached for $p_{L}=0.2$, where the throughput is almost doubled with respect to the no RG case. Clearly, this is due to the exploitation of RG leaders which provide coverage to nodes that would be otherwise disconnected from all APs. As the node density grows larger than 0.004 users per unit area (i.e., per square meter), relaying causes too much interference among parallel transmissions and the interference effect dominates over connectivity improvements.

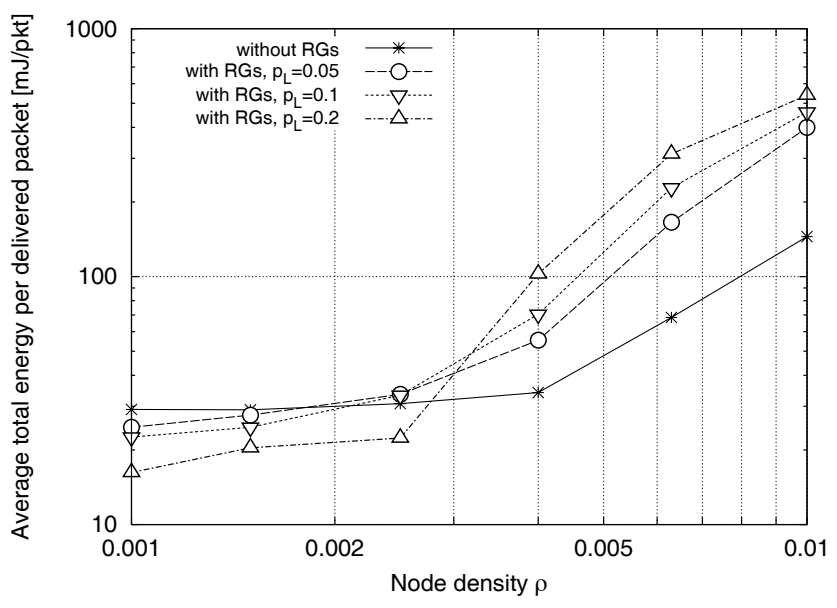

Fig. 3. Average energy consumed to delivery a packet as a function of the node density $\rho$.

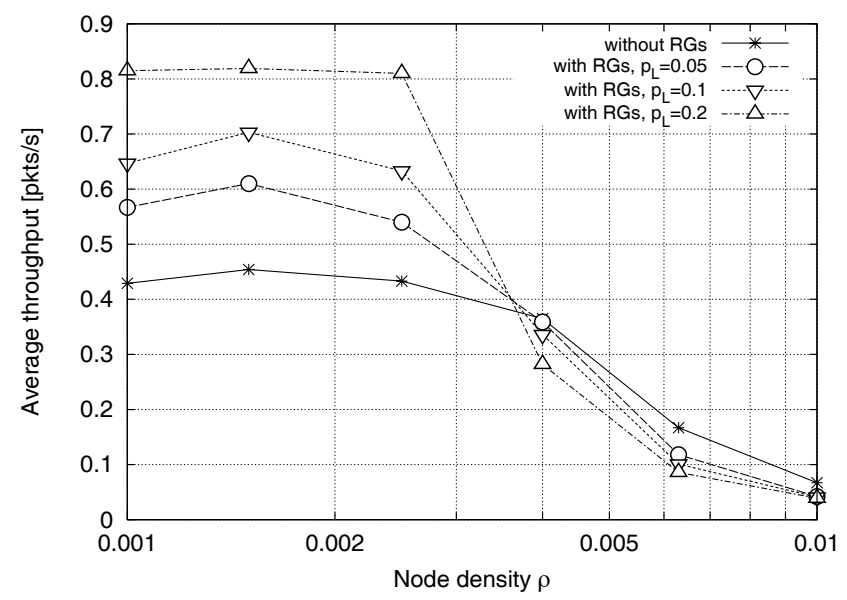

Fig. 4. Average network throughput as a function of the node density $\rho$.

The obtained results verify the correctness of the analytical method. Even though the analysis does not take into consideration some implementation aspects, the qualitative behavior is similar.

However, the simulation approach is also useful as it is able to correctly model multi-user interference, which would cause excessive mathematical complexity. Interference issues also justify the disagreement between the results obtained with the two evaluation methods when the node density exceeds a certain level: simulation results show the congestion due to interference, while this is not reflected in the analysis.

\section{CONCLUSions}

This letter focused on the performance evaluation of the use of Routing Groups (RGs) in Ambient Networks. We studied the improvements in terms of decreased signaling overhead and system interference given by RGs. Both an accurate simulation tool and a simple yet effective analytical model were discussed.

The theoretical approach correctly evaluates the general trade-offs in RG establishment. However, accurate quantitative evaluation of RG algorithms can only be obtained through detailed simulation. The full dynamics at the physical layer 
are, in fact, difficult to track by analysis. Neglecting them, as observed in our results, often leads to performance bounds which may hide important behaviors.

\section{REFERENCES}

[1] The Ambient Networks Project, http://www.ambient-networks.org/.

[2] C.-K. Toh, "Associativity-based routing for ad hoc mobile networks," Kluwer Wireless Personal Commun., vol. 4, no. 2, pp. 103-139, Mar. 1997.

[3] C. Prehofer and B. Souville, "Synchronized reconfiguration of a group of mobile nodes in ad-hoc networks," in Proc. IEEE ICT 2003, vol. 1, Tahiti, Papeete, French Polynesia, Feb. 2003, pp. 400-405.

[4] P. Popovski, F. H. P. Fitzek, H. Yomo, T. K. Madsen, R. Prasad, and N. J. Vej, "MAC-layer approach for cluster-based aggregation in sensor networks," in Proc. International Workshop on Wireless Ad-Hoc Networks (IWWAN), Oulu, Finland, 2004, pp. 89-93.

[5] M. Chatterjee, S. K. Das, and D. Turgut, "WCA: a weighted clustering algorithm for mobile ad hoc networks," Kluwer Cluster Computing, vol. 5, no. 2, pp. 193-204, Apr. 2002.

[6] S. Basagni, "Distributed and mobility-adaptive clustering for multimedia support in multi-hop wireless networks," in Proc. IEEE VTC Fall, vol. 2, Amsterdam, The Netherlands, Nov. 1999, pp. 889-893.

[7] M. Zonoozi and P. Dassanayake, "User mobility modeling and characterization of mobility patterns," IEEE J. Select. Areas Commun., vol. 15, no. 7 , pp. $1239-1252$, Sept. 1997.
[8] K. Xu and M. Gerla, "A heterogenous routing protocol based on a new stable clustering scheme," in Proc. IEEE MILCOM, vol. 2, Oct 2002, pp. 838-843.

[9] M. Rossi, L. Badia, P. Giacon, and M. Zorzi, "On the effectiveness of logical device aggregation in multi-radio multi-hop networks," in Proc. IEEE WIRELESSCOM, vol. 1, June 2005, pp. 354-361.

[10] A. Goldsmith, Wireless Communications. Cambridge University Press, 2005.

[11] W. C. Jakes, Microwave Mobile Communications. Wiley-IEEE Press, 1994.

[12] H. Holma and A. Toskala, WCDMA for UMTS: Radio Access for Third Generation Mobile Communication, 3rd ed. John Wiley \& Sons Ltd, 2004.

[13] IEEE 802 LAN/MAN Standards Committee documentation, http://www.ieee802.org.

[14] M. Rossi, N. Bui, L. Badia, and M. Zorzi, "On group mobility patterns and their exploitation to logically aggregate terminals in wireless networks," in Proc. IEEE VTC Fall, vol. 3, Dallas, TX, US, Sept. 2005, pp. $1863-1867$.

[15] B. McDonald and T. Znati, "Comparative analysis of neighbor greeting protocols: ARP versus ES-IS," in Proc. IEEE SIMULATION, New Orleans, LA, US, Apr. 1996, pp. 71-80. 